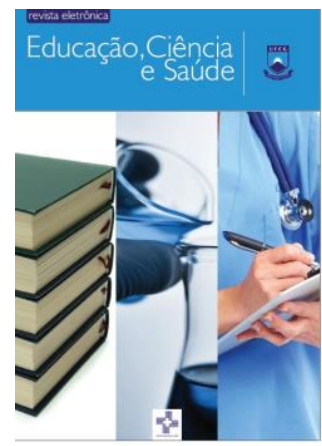

EDUCAÇÃO CIÊNCIA E SAÚDE

http://dx.doi.org/10.20438/ecs.v5i1.155

\title{
ESTUDO ANALÍTICO DO PROCESSO DE SECAGEM DE TELHAS CERÂMICAS USANDO MODELOS DE DIFUSÃO UNIDIMENSIONAL
}

\author{
Helymarckson Batista de Azevedo ${ }^{1}$, Willian Oliveira Santos ${ }^{1}$, Isaac Ferreira de \\ Lima $^{1}$, Vera Solange de Oliveira Farias², Célia Maria Rufino Franco², Paula \\ Isabella de Oliveira Rocha ${ }^{3}$ \\ ${ }^{1}$ Alunos do curso de Licenciatura em Física e Matemática, Unidade Acadêmica de Física e \\ Matemática, Universidade Federal de Campina Grande, Cuité-PB, Brasil. \\ ${ }^{2}$ Prof. Unidade Acadêmica de Física e Matemática, Universidade Federal de Campina Grande, \\ Cuité, PB, Brasil. \\ ${ }^{3}$ Curso de graduação em Engenharia de Civil- CTRN, Universidade Federal de Campina \\ Grande, Campina Grande-PB, Brasil. \\ Email para correspondência: helymarcksonazevedo@yahoo.com.br
}

\begin{abstract}
Resumo
A secagem é um processo amplamente utilizado em diversos setores industriais, entre eles a indústria de materiais cerâmicos. O objetivo deste trabalho é utilizar o modelo da difusão líquida para descrever a secagem de telhas cerâmicas do tipo canal, aproximando sua geometria por uma parede infinita. Procedimentos experimentais de secagem de amostras do produto com teor de umidade inicial de aproximadamente 0,20 (b.s.) foram analisados para duas temperaturas distintas, 60 e $70^{\circ} \mathrm{C}$. A solução da equação de difusão de massa unidimensional foi obtida para duas condições de contorno (prescrita e convectiva). As propriedades termofísicas do produto foram determinadas através de otimizadores acoplados à solução analítica (método inverso). Os resultados mostraram que o modelo proposto descreve de forma satisfatória o processo de secagem e que os valores obtidos para os parâmetros termofísicos são concordantes com outros trabalhos presentes na literatura para estudos similares. Foi observado ainda que, a condição de contorno convectiva é a mais apropriada para descrever o processo.
\end{abstract}

Palavras-chave: difusividade de massa, otimização, simulação, cinética de secagem.

\begin{abstract}
Drying is a widely used process in several industrial sectors, among them the ceramic materials industry. The purpose of this work is to use the model of the liquid diffusion to describe the drying of ceramic tiles of the canal type, approaching its geometry to an infinite wall. Experimental drying procedures for product samples with an initial moisture content of approximately 0.20 (d.b.) were analyzed for two different temperatures, 60 and $70^{\circ} \mathrm{C}$. The solution of the one-dimensional mass diffusion equation was obtained for two boundary conditions (prescribed and
\end{abstract}


convective). The thermophysical properties of the product were determined through optimizers coupled with the analytical solution (inverse method). The results showed that the proposed model describes satisfactorily the drying process and that the values obtained for the thermophysical parameters are in agreement with other works present in the literature for similar studies. It was also observed that the convective boundary condition is the most appropriate to describe the process.

Keywords: mass diffusivity, optimization, simulation, kinetics of drying.

\section{Introdução}

Uma importante etapa em vários processos produtivos, nos mais diversos setores da atividade humana, refere-se à secagem de produtos úmidos, sendo amplamente aplicada nas indústrias agrícolas, cerâmicas, químicas, alimentícias, farmacêuticas, de papel e celulose, mineral e de polímeros (PARK et al., 2007). Dentre as várias áreas, uma extremamente importante, na qual a secagem é uma das etapas fundamentais do processo produtivo, é aquela referente à fabricação de materiais cerâmicos (blocos, tijolos, telhas, revestimentos e pisos) que são amplamente utilizados na construção civil. Os produtos de cerâmica vermelha têm como principal matéria prima a argila que, ao ser misturada com certa quantidade de água, forma uma massa coesiva que pode ser moldada com facilidade. Esta propriedade é característica dos minerais argilosos e denomina-se plasticidade. A quantidade de água a ser adicionada deve ser controlada e retirada antes da queima do produto, para que este não seja danificado (BASTOS, 2003; BACCELLI JÚNIOR, 2010; PERDONÁ, 2012). A remoção da água se dá através do processo de secagem que é uma etapa bastante delicada e complexa no processo de fabricação de cerâmica vermelha.

A descrição do fenômeno de secagem pode ser feita pela utilização dos chamados mecanismos de migração de massa que são descritos pela literatura. Dentre esses mecanismos, destaca-se o da difusão líquida, que tem sido amplamente utilizado por pesquisadores em fenômenos que envolvam transferência de massa (CHEMKHI et al., 2005; FARIAS et al., 2009; FARIAS et al., 2010; SILVA et al., 2012; SILVA et al., 2013).

A taxa de perda de umidade durante a secagem é um fator importante no controle do processo, pois afeta as propriedades finais dos produtos (SILVA et al., 2013). Em geral, os processos de secagem para materiais cerâmicos podem ser divididos em dois períodos. O primeiro período de secagem também 
é conhecido como período de taxa constante, uma vez que a variação do teor de umidade por unidade de tempo permanece estável. O segundo período é chamado período de taxa de secagem decrescente e termina quando o sólido atinge o teor de umidade de equilíbrio com o meio circundante. $O$ modelo de difusão não descreve bem o período de taxa constante, sendo aplicado a partir do início do período de taxa de secagem decrescente (DINCER e DOST, 1995; DONDI, 2014; FARIAS et al., 2018).

$\mathrm{Na}$ literatura existem alguns trabalhos sobre secagem de produtos cerâmicos (placas e telhas cerâmicas) e que usam o modelo difusivo para descrição do processo (SANDER et al., 2003; CHEMKHI e ZAGROUBA, 2005; SILVA et al., 2013; FARIAS et al., 2013; FARIAS et al. 2015; LIMA, 2016; FARIAS et al., 2018).

Farias et al. (2018) apresentaram estudos sobre a secagem de telhas cerâmicas variando a temperatura e/ou a velocidade do ar de secagem e calcularam os parâmetros termofísicos, a partir das curvas de secagem. A condição de contorno usada foi a de terceiro tipo e os resultados obtidos foram compatíveis com dados reportados na literatura para pesquisas similares.

Diante do exposto, o objetivo principal deste artigo é descrever a secagem de telhas cerâmicas durante o período da taxa de secagem decrescente, usando modelos de difusão unidimensional, incluindo otimização e simulação do processo. Assim, otimizadores acoplados às soluções analíticas unidimensionais com condição de contorno prescrita e convectiva foram utilizados para obter os parâmetros termofísicos e em seguida simular as cinéticas de secagem.

\section{Materiais e métodos}

O modelo difusivo é expresso pela equaçao da difusão. Neste trabalho, soluções desta equação para a geometria parede infinita serão utilizadas, empregando duas condições de contorno do problema (prescrita e convectiva). A seguir serão mostradas as soluções utilizadas.

\subsection{Equação da Difusão de Massa}

A equação de difusão de massa pode ser expressa da seguinte forma: 


$$
\frac{\partial}{\partial \mathrm{t}}(\mathrm{M})=\nabla \cdot(\mathrm{D} \nabla \mathrm{M})
$$

onde $\mathrm{M}$ é a função espaço-temporal para $\mathrm{o}$ teor de umidade e $\mathrm{D}$ é a difusividade efetiva de massa.

Para região de uma parede infinita, a equação da difusão de massa, em coordenadas cartesianas e considerando regime transiente (variação de $\mathrm{M}$ com o tempo) é escrita na forma unidimensional como mostra a equação a seguir:

$$
\frac{\partial \mathrm{M}}{\partial \mathrm{t}}=\frac{\partial}{\partial \mathrm{x}}\left(\mathrm{D} \frac{\partial \mathrm{M}}{\partial \mathrm{x}}\right) \text {. }
$$

A Equação (2) pode ser resolvida numericamente, caso a variação dos parâmetros de transporte como a difusividade (D) e/ou das dimensões do corpo sejam consideradas durante a secagem. Caso as variações de $D$ e das dimensões do sólido não sejam levadas em consideração durante o processo, uma solução analítica pode ser utilizada.

Neste trabalho, optou-se por utilizar-se de soluções analíticas da Equação (2), obtidas por separação de variáveis. No entanto, para utilização desta solução em um problema real, algumas hipóteses devem ser consideradas, que são:

- As dimensões do sólido não variam durante a difusão da água;

- A difusão líquida é o único mecanismo de transporte de água dentro do sólido;

- A distribuição inicial do teor de umidade deve ser uniforme;

- O sólido é considerado homogêneo e isotrópico;

- Os parâmetros de transporte não variam durante o processo.

Para resolver a Equação (2) são necessárias condições iniciais e de contorno. As condições de contorno aplicadas foram de primeira e terceira espécies, como apresentado a seguir.

\subsubsection{Solução da Equação de difusão utilizando condição de contorno de primeira espécie}

Numa parede infinita, considera-se apenas o fluxo de uma grandeza através de sua espessura Lx (Figura 1), pois as dimensões de sua largura Ly e de seu comprimento Lz são muito grandes em comparação com Lx, de forma que os fluxos da grandeza tratada nas direções y e z podem ser desconsiderados. 
Sendo o domínio da parede de -Lx/2 até $L x / 2$ (considera-se o centro de simetria do corpo como sendo a origem de um sistema de coordenadas) e aplicando as condições de contorno e simetria de primeira espécie na solução da Equação (2) obtida por separação de variáveis obtém-se que a solução da equação de difusão de massa para uma parede infinita em regime estacionário é dada pela expressão (LUIKOV; 1968; CRANK, 1992; BIRD et al, 2001; INCROPERA et al, 2008):

$$
M(x, t)=M_{e q}+\left(M_{i}-M_{e q}\right) \sum_{n=1}^{\infty} C_{n} \cos \left(\mu_{n} \frac{x}{L x / 2}\right) \exp \left[-\mu_{n}^{2} \cdot \frac{D}{\left(\frac{L x}{2}\right)^{2}} t\right]
$$

onde:

$$
\mu_{\mathrm{n}}=\frac{(2 \mathrm{n}-1) \pi}{2} \text { e } \mathrm{C}_{\mathrm{n}}=\frac{2(-1)^{\mathrm{n}+1}}{\mu_{\mathrm{n}}}
$$

Sendo que L é a espessura da parede infinita.
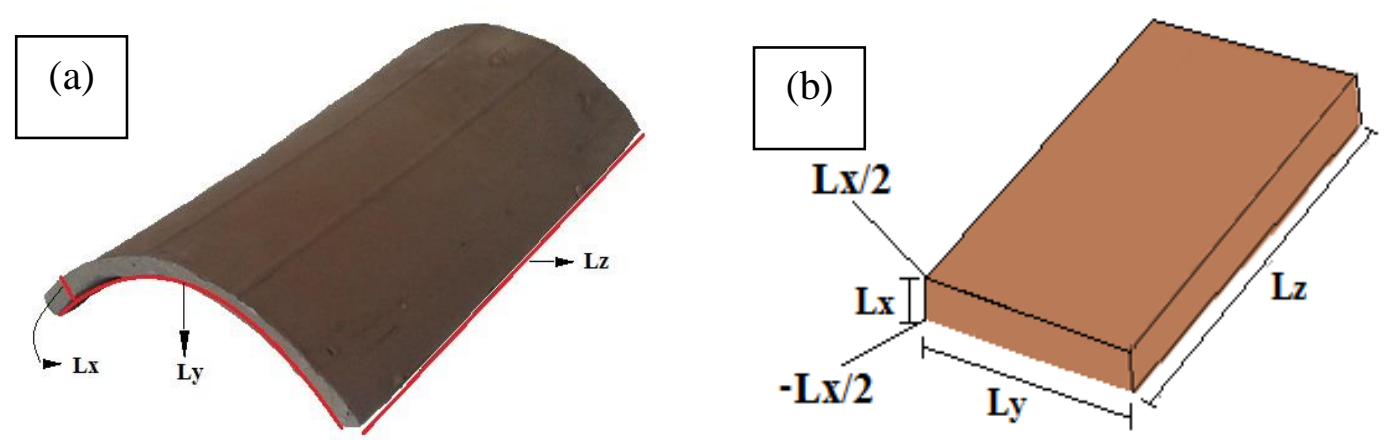

Figura 1: (a) Telha utilizada na secagem; (b) Geometria aproximada da telha para uma parede infinita.

A Equação (3) pode fornecer o valor de $M$ para qualquer instante e posição conhecido. O valor médio da grandeza de interesse (M) é dado por:

$$
\bar{M}(t)=M_{e q}+\left(M_{i}-M_{e q}\right) \sum_{n=1}^{\infty} \frac{8}{(2 n-1)^{2} \pi^{2}} \exp \left[-\mu_{n}^{2}-\frac{D}{\left(\frac{L x}{2}\right)^{2}} t\right] \text {. }
$$




\subsubsection{Solução da equação de difusão utilizando condição de contorno de terceiro tipo}

A condição de contorno de terceira espécie, conhecida também como convectiva se caracteriza por igualar na superfície do sólido os fluxos da grandeza de interesse por meio de condução e convecção (FARIAS, 2011; SILVA et al., 2012; FARIAS et al., 2015). Esta condição de contorno considera que existe uma certa resistência para que a superfície do sólido atinja a situação de equilíbrio com o ambiente. Desta forma, o fluxo convectivo na superfície externa do material deve ser igual ao fluxo difusivo no interior do material. Para a parede infinita, esta igualdade é dada por:

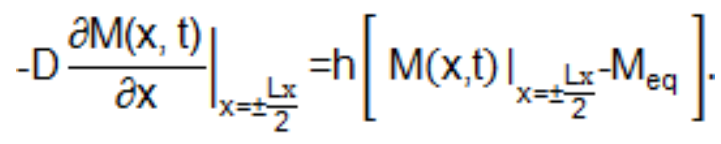

Aplicando a primeira condição de contorno de simetria $\left(\left.\frac{\partial \mathrm{M}}{\partial \mathrm{x}}\right|_{\mathrm{x}=0}=0\right) \mathrm{e}$, posteriormente a condição de contorno explícita na Equação (5) na solução da Equação (2), tem-se que (LUIKOV; 1968; CRANK, 1992; BIRD et al, 2001; INCROPERA et al, 2008):

$$
M\left(x_{t} t\right)=M_{e q}-\left(M_{e q}-M_{i}\right)\left[\sum_{n=1}^{\infty} A_{n} \cos \left(\mu_{n} \frac{x}{\frac{L x}{2}}\right)\right] \times \exp \left[-\left(\frac{\mu_{n}}{\frac{L x}{2}}\right)^{2} D t\right],
$$

onde:

$$
A_{n}=\frac{4 \operatorname{sen}\left(\mu_{n}\right)}{2 \mu_{n}+\operatorname{sen}\left(2 \mu_{n}\right)^{\prime}}
$$

e $\mu_{\mathrm{n}}$ são as raízes da equação característica para a parede infinita, dada por:

$$
\cot \mu_{n}=\frac{\mu_{n}}{B i^{x}}
$$

O parâmetro Bi (número de Biot) é definido da seguinte forma:

$$
\mathrm{Bi}=\frac{\mathrm{hLx} / 2}{\mathrm{D}}
$$

(razão entre o efeito convectivo e o difusivo), e Lx é o comprimento característico do produto.

O valor médio de $\mathrm{M}$, para esta condição de contorno, é dado por: 


$$
\bar{M}(t)=M_{e q}-\left(M_{e q}-M_{i}\right) \sum_{n=1}^{\infty} B_{n} \exp \left[-\left(\frac{\mu_{n}}{L x}\right)^{2} D t\right],
$$

sendo coeficiente $B_{n}$ dado por:

$$
\mathrm{B}_{\mathrm{n}}=\frac{2 \mathrm{Bi}^{2}}{\left(\mu_{\mathrm{n}}\right)^{2}\left(\mathrm{bi}^{2}+\mathrm{Bi}+\mu_{\mathrm{n}}\right)^{\prime \prime}}
$$

onde os termos $\mu_{n}$ e $B_{i}$ são relacionados pela Equação (8), que é uma equação transcendental.

\subsection{Otimização: Determinação de Parâmetros}

Para determinar a difusividade efetiva de água (D) no sólido e o coeficiente convectivo de transferência de massa (h) foram empregados os softwares: Prescribed Adsorption-Desorption (SILVA e SILVA, 2008), para a solução com condição de contorno de primeiro tipo e o Convective Adsorption-Desorption (SILVA e SILVA, 2009a) para a solução com condição de contorno de terceiro tipo.

Os softwares apresentam interface simples, sendo necessário carregar o conjunto de pontos experimentais obtidos na secagem (teor de umidade, tempo) num arquivo texto. Posteriormente, o software disponibiliza as geometrias para as quais o otimizador tem solução. As geometrias são: parede infinita, cilindro infinito, esfera, cilindro finito e paralelepípedo. Após a escolha da geometria, os programas solicitam que sejam colocadas a(s) dimensão(ões) do sólido e os teores de umidade inicial $\left(M_{i}\right)$ e de equilíbrio $\left(M_{\text {eq }}\right)$ obtidos através da secagem.

O Prescribed e o Convective são acoplados às soluções analíticas da equação da difusão de massa para uma geometria de parede infinita pressupondo condições de contorno de primeira e terceira espécies, respectivamente (Equações (4) e (10)). Estes otimizadores determinam os parâmetros de transporte minimizando a função qui-quadrado $\left(\chi^{2}\right)$. O quiquadrado é dado por:

$$
\mathrm{X}^{2}=\sum_{\mathrm{i}=1}^{\mathrm{N}_{\mathrm{p}}}\left[\overline{\mathrm{M}}^{\mathrm{exp}}-\overline{\mathrm{M}}^{\mathrm{ana}}\left(\mathrm{D}_{v}, \mathrm{Bi}_{1}\right)\right]^{2} \frac{1}{\sigma_{i}^{2}}
$$


onde Np é o número de dados experimentais, $\overline{\mathrm{M}}^{\exp }$ e $\overline{\mathrm{M}}^{\mathrm{ana}}{ }^{\mathrm{a}}$ são os valores do teor de umidade médio experimentais e simulados, respectivamente. O termo $\mathrm{Bi}_{1}$ é considerado na solução com condição de contorno de terceiro tipo, sendo tomado como infinito para a condição de contorno de primeiro tipo. O peso estatístico $\left(\frac{1}{\sigma_{i}^{2}}\right)$ é o peso estatístico do i-ésimo ponto experimental e, geralmente é dado como sendo igual a unidade.

\subsection{Procedimento Experimental}

$O$ produto submetido à secagem neste trabalho foram telhas do tipo canal oriundas de uma olaria (Cerâmica Barros) da cidade de Picuí-PB. As telhas foram recolhidas na olaria após sua conformação e acondicionadas (úmidas) em sacos plásticos para evitar a perda de água.

Os corpos de prova foram conduzidos até o laboratório de Bromatologia da Universidade Federal de Campina Grande, campus Cuité-PB, para serem secas numa estufa de ventilação e circulação de ar da marca AMERICAN LAB modelo AL 102/480. No referido laboratório, os corpos de prova a serem secos tiveram suas dimensões e massas medidos antes de ser iniciado o processo de secagem.

A massa foi medida por uma balança da marca BIOPRECISA modelo JH2102 com medição de valor máximo de massa igual a 2100,00 gramas. As dimensões foram medidas por um paquímetro universal da marca PONTEC de precisão de 0,05 milímetros e por uma trena da marca BRASFORTE.

Foram realizadas duas secagens em dias distintos e com duas temperaturas distintas: A primeira a $70^{\circ} \mathrm{C}$ e a segunda secagem realizada a uma temperatura de $60^{\circ} \mathrm{C}$. Os valores dos teores de umidade inicial $\left(\mathrm{M}_{\mathrm{i}}\right)$ e de equilíbrio $\left(M_{\text {eq }}\right)$ em base seca (b.s.), bem como as dimensões das telhas são mostradas na Tabela 1.

Tabela 1: Medidas das dimensões e das massas da telhas para cada secagem.

\begin{tabular}{|c|c|c|c|c|c|}
\hline $\mathrm{T}\left({ }^{\circ} \mathrm{C}\right)$ & $\mathrm{M}_{\mathrm{i}}$ (b.s.) & $\mathrm{M}_{\mathrm{eq}}$ (b.s.) & $\mathrm{Lx}(\mathrm{mm})$ & $\mathrm{Ly}(\mathrm{mm})$ & $\mathrm{Lz}(\mathrm{mm})$ \\
\hline 60 & 0,1978 & 0,0139 & 8,20 & 141,6 & 295,5 \\
\hline 70 & 0,1944 & 0,0094 & 9,20 & 125,90 & 284,00 \\
\hline
\end{tabular}


onde Lx é a espessura, Ly a largura e Lz o comprimento da telha.

As massas das telhas foram medidas durante as secagens em instantes pré-determinados. As secagens ocorreram até que a variação das massas das telhas pudesse ser desconsiderada (variação de miligramas por unidade de tempo). A última massa medida nesta etapa corresponde à massa de equilíbrio, usada para determinação do teor de umidade de equilíbrio. Após a secagem, a estufa foi colocada a uma temperatura de $105^{\circ} \mathrm{C}$ para que as telhas utilizadas nos dois procedimentos fossem recolocadas e toda sua umidade fosse retirada e, assim fosse possível medir a massa seca das telhas (sem umidade).

Foi realizada ainda, uma caracterização granulométrica e química da argila utilizada na fabricação das telhas no Laboratório de Caracterização de Materiais da Universidade Federal de Campina Grande Campus Campina Grande-PB. O histograma da distribuição granulométrica da argila é apresentado na Figura 2.

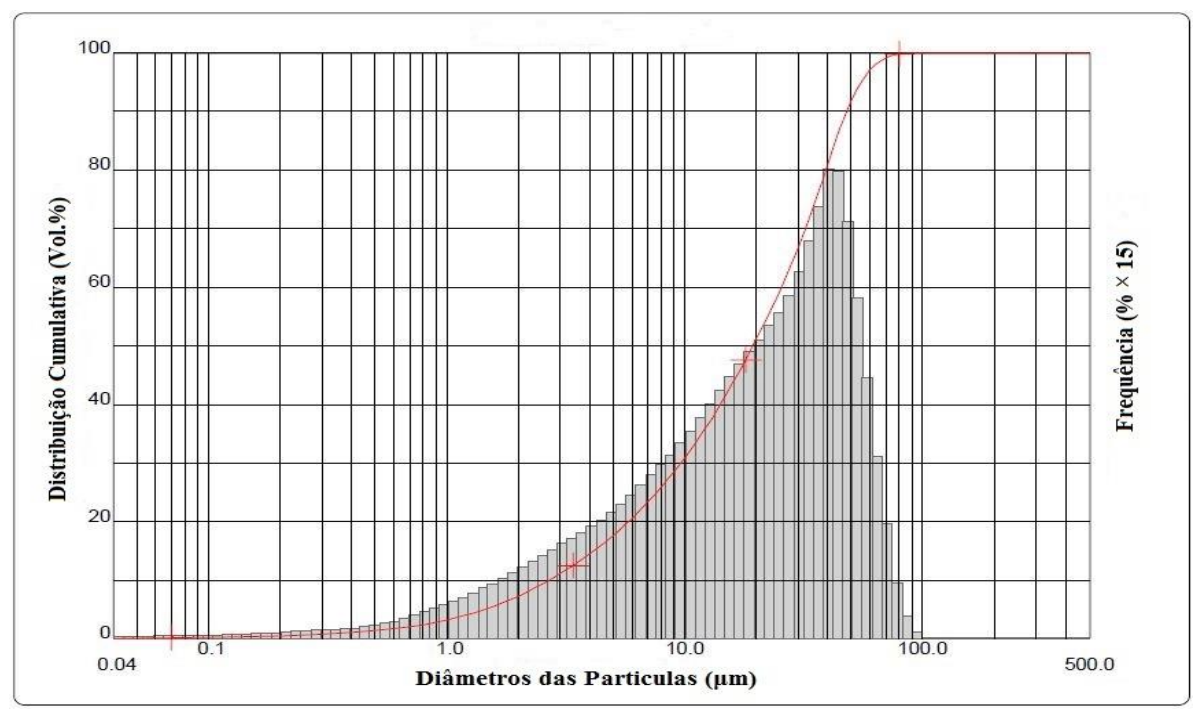

Figura 2: Distribuição granulométrica da argila e curva cumulativa.

$\mathrm{Na}$ caracterização granulométrica da argila foram medidos os tamanhos das partículas que a argila é composta. A Figura 2 mostra que as partículas têm tamanhos variando de 1 (um) a 100 (cem) micrometros ( $\mu \mathrm{m}$ ) com $\mathrm{D}_{10}$ de 2,70 $\mu \mathrm{m}, \mathrm{D}_{50}$ de 19,36 $\mu \mathrm{m}$, $\mathrm{D}_{90}$ de 48,01 $\mu \mathrm{m}$ e um diâmetro médio de 22,92 $\mu \mathrm{m}$.

A Tabela 2 mostra a composição química da argila e o respectivo percentual presente na amostra. A argila apresenta uma constituição 
principalmente de sílica ( $\mathrm{SiO} 2)$, alumínio (AI2O3) e ferro $\left(\mathrm{Fe}_{2} \mathrm{O}_{3}\right)$, possuindo outros componentes em menor percentual, como o potássio $\left(\mathrm{K}_{2}\right)$, cálcio $(\mathrm{Ca})$ e titânio (Ti).

Tabela 2: Composição química da argila que compõe as telhas utilizadas.

\begin{tabular}{|c|c|c|c|c|c|c|c|c|}
\hline Componentes & $\mathrm{SiO} 2$ & $\mathrm{~A} 12 \mathrm{O} 3$ & $\mathrm{Fe}_{2} \mathrm{O}_{3}$ & $\mathrm{MgO}$ & $\mathrm{K} 2 \mathrm{O}$ & $\mathrm{CaO}$ & $\mathrm{TiO}_{2}$ & Outros \\
\hline $\begin{array}{c}\text { Composição } \\
\text { Química (\%) }\end{array}$ & 55,20 & 22,49 & 11,21 & 3,53 & 3,41 & 1,91 & 1,13 & 1,11 \\
\hline
\end{tabular}

A seguir serão apresentados os resultados obtidos com a metodologia descrita anteriormente e aplicada nas secagens de telhas.

\section{$3 \quad$ Resultados e Discussão}

Com os valores das massas das telhas para cada instante de tempo, obtidos durante as secagens, determinou-se o teor de umidade em base seca (b.s) para tais instantes. A determinação da difusividade de massa (D) necessita desta associação entre o teor de umidade com o respectivo tempo de secagem, visto que o método inverso faz uma comparação entre os valores experimentais e os simulados pelos softwares, buscando a melhor aproximação para descrição do processo.

Com os valores do teor de umidade determinados, foi possível plotar o gráfico da cinética de secagem para as duas temperaturas do processo. Os gráficos são apresentados na Figura 3, onde é mostrada a ocorrência nos dois casos da presença dos dois períodos de secagem: taxa constante do início até cerca de sessenta (60) minutos para secagem a $70{ }^{\circ} \mathrm{C}$ e sessenta e cinco (65) minutos para secagem a $60^{\circ} \mathrm{C}$; seguidos de um período de secagem com taxa decrescente até o final do processo. Para ambos os processos, o período de taxa decrescente inicia-se quando o teor de umidade é aproximadamente 0,12 em base seca.

Como já foi dito, o modelo difusivo descreve apropriadamente apenas o período de taxa decrescente (SILVA et al., 2013; FARIAS et al., 2013; FARIAS et al., 2015).

Os gráficos do teor de umidade em função do tempo para as duas secagens foram plotados através do software LAB Fit Curve Fit Software (SILVA e SILVA, 2009b). 
Os dados experimentais obtidos mostraram que o período de taxa constante ocorre no início do processo de secagem das telhas (geralmente entre os sessenta (60) primeiros minutos da secagem), como mostra a Figura 3. Posteriormente a este período, verifica-se um período de taxa de secagem decrescente.
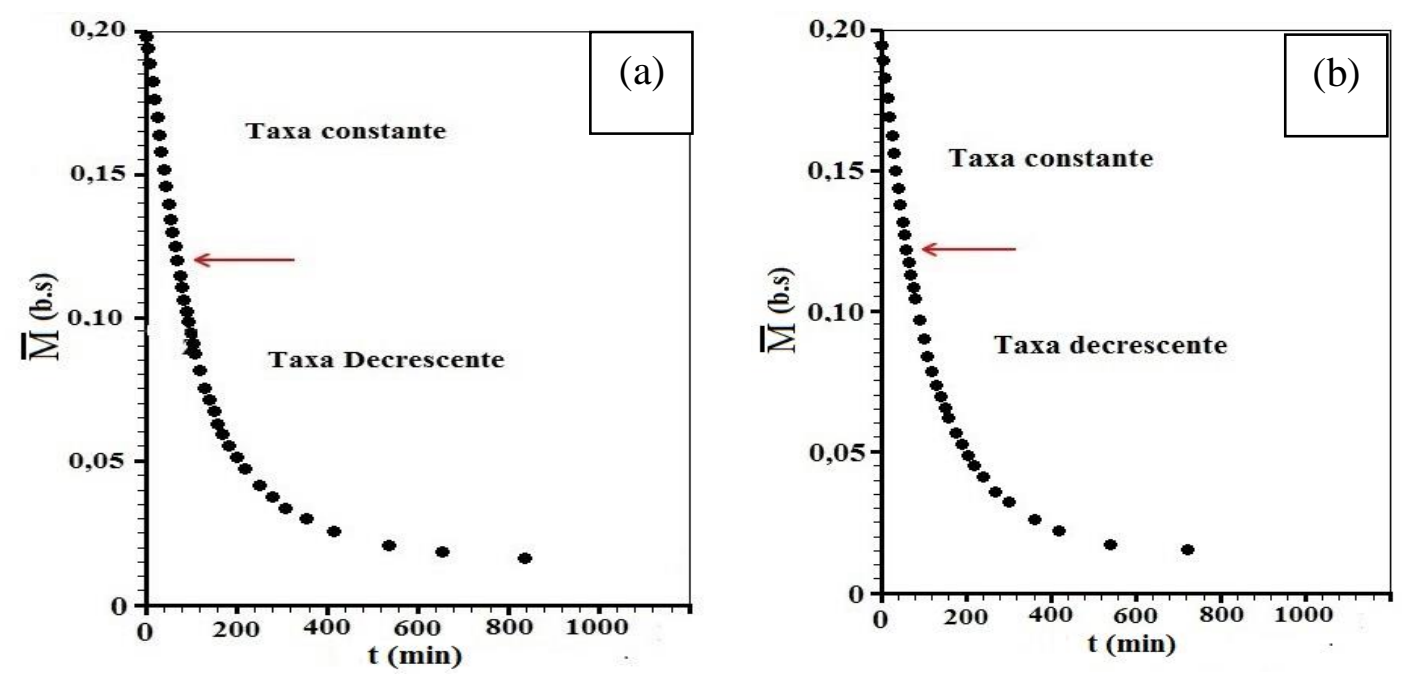

Figura 3: Gráficos com os pontos experimentais da secagem, sendo indicados os períodos de taxa de secagem constante e taxa decrescente nas secagens a temperatura de: (a) $60^{\circ} \mathrm{C}$; (b) $70^{\circ} \mathrm{C}$.

A Figura 4 apresenta os gráficos da taxa de secagem no período constante para as duas temperaturas. Os gráficos são retas (equação linear) de equação $M=A t+B$. Os coeficientes $A$ e $B$ são mostrados na Figura 4 e foram obtidos por uma regressão linear no LAB Fit Curve Fit Software (SILVA e SILVA, 2009b). 

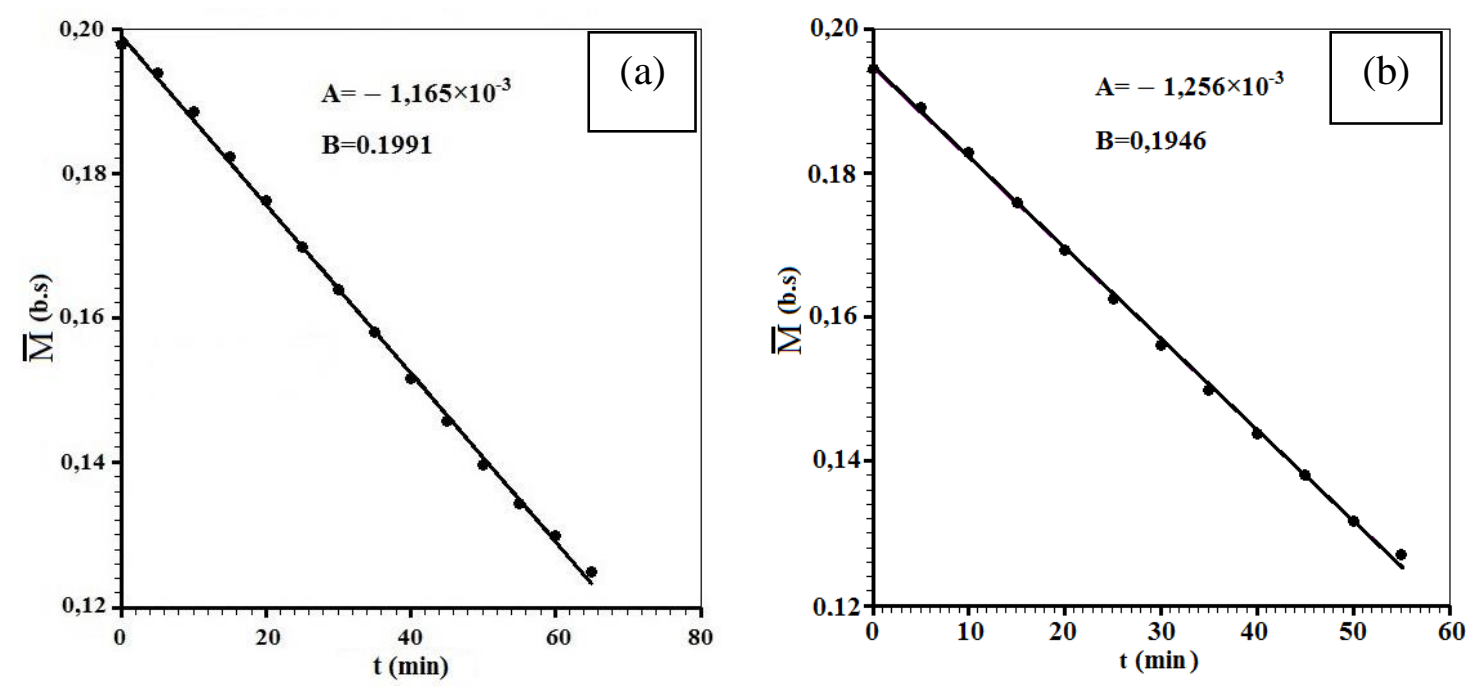

Figura 4: Gráficos da cinética de secagem do período de taxa constante para as secagens: (a) a $60^{\circ} \mathrm{C}$; (b) a $70^{\circ} \mathrm{C}$.

Excluindo o período de taxa constante para as duas temperaturas, os dados experimentais $(\mathrm{M}, \mathrm{t})$, bem como as dimensões da telha e os teores de umidade inicial e de equilíbrio foram carregados nos softwares Prescribed e Convective. Em seguida foi iniciado o processo de otimização para calcular os parâmetros do processo para a parede infinita.

O software Prescribed é um otimizador de condição de contorno de equilíbrio, logo disponibiliza a difusividade efetiva de massa e os indicadores estatísticos $\mathrm{R}^{2}$ e o qui-quadrado $\left(\chi^{2}\right)$, de forma que quanto mais próximo de um (1) for $\circ \mathrm{R}^{2}$ e menor for o $\chi^{2}$, melhor é o resultado da simulação. A simulação realizada a partir dos dados experimentais obtidos na secagem das telhas usando o software Prescribed forneceu os resultados expressos na Tabela 3.

Tabela 3: Resultados disponibilizados para $D$ pelo software Prescribed.

\begin{tabular}{|c|c|c|c|}
\hline $\mathrm{T}\left({ }^{\circ} \mathrm{C}\right)$ & $\mathrm{D}\left(\mathrm{m}^{2} \min ^{-1}\right)$ & $\mathrm{R}^{2}$ & $\chi^{2}$ \\
\hline 60 & $3.923 \times 10^{-8}$ & 0.98998 & $4.6426 \times 10^{-2}$ \\
\hline 70 & $4.545 \times 10^{-8}$ & 0.99038 & $3,9951 \times 10^{-2}$ \\
\hline
\end{tabular}

O otimizador acoplado com a solução de equação de difusão de massa com condição de contorno de terceira espécie (Convective) faz uma simulação considerando a igualdade dos fluxos convectivo e difusivo na superfície do corpo a ser seco. Logo, esta simulação disponibiliza a difusividade efetiva de massa do sólido (D), assim como o coeficiente de transferência convectivo de 
massa (h) e o número de Biot para o qual o somatório de soluções tem os melhores indicadores estatísticos.

Após serem realizadas as otimizações o software Convective apresentou os resultados expressos na Tabela 4:

Tabela 4: Resultados para D, h e o número de Biot pelo software Convective.

\begin{tabular}{|c|c|c|c|c|c|}
\hline $\mathrm{T}\left({ }^{\circ} \mathrm{C}\right)$ & $\mathrm{D}\left(\mathrm{m}^{2} / \mathrm{min}\right)$ & $\mathrm{h}(\mathrm{m} / \mathrm{min})$ & $\mathrm{R}^{2}$ & $\chi^{2}$ & Biot \\
\hline 60 & $7,526 \times 10^{-8}$ & $7,572 \times 10^{-5}$ & 0,9971 & $9,6383 \times 10^{-3}$ & 4,1250 \\
\hline 70 & $8,856 \times 10^{-8}$ & $7,460 \times 10^{-5}$ & 0,9976 & $6,1436 \times 10^{-3}$ & 3,8750 \\
\hline
\end{tabular}

Os softwares Prescribed e Convective ainda disponibilizam os gráficos em que os pontos experimentais e a curva de secagem (gerada pela simulação que os programas realizam, mediante carregamento dos dados reais da secagem) são sobrepostos.

Os gráficos gerados pelo programa Prescribed para as duas simulações realizadas estão ilustrados na Figura 5.
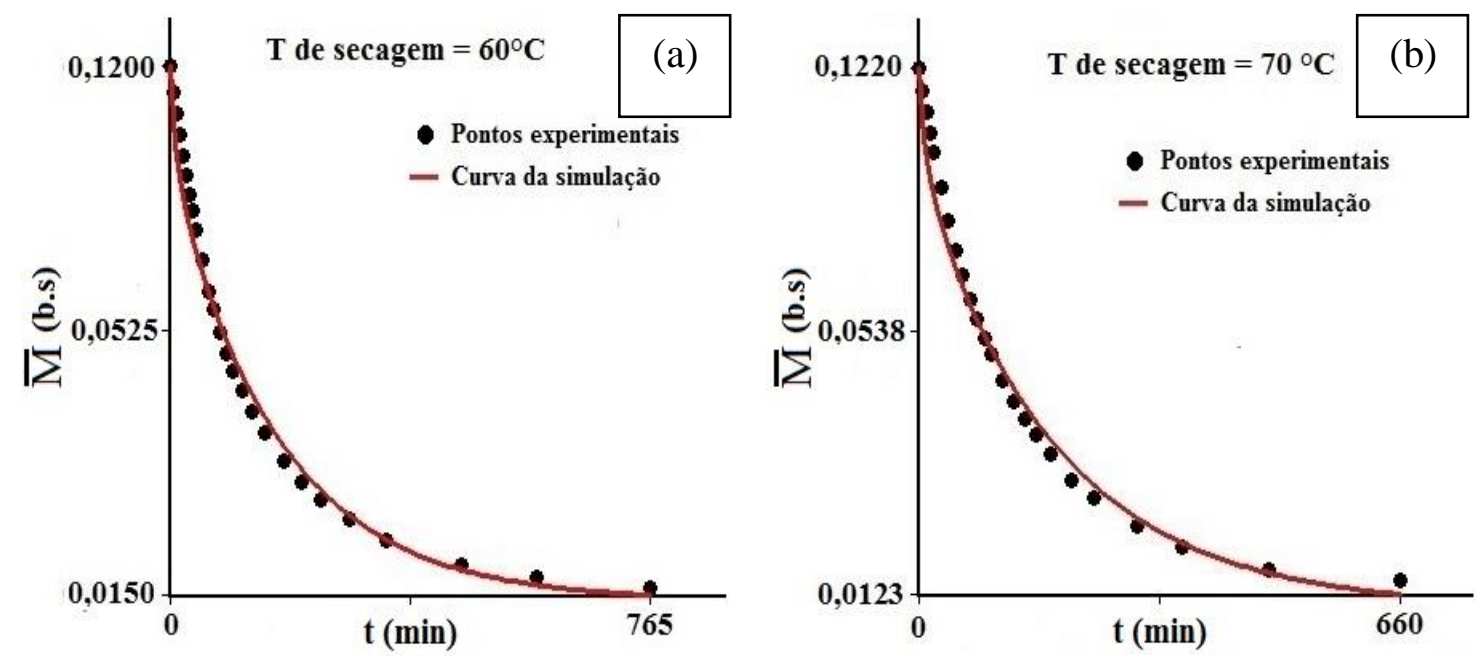

Figura 5: Gráficos das cinéticas de secagem para os períodos de taxa decrescente com pontos experimentais e a curva de secagem simulada pelo software Prescribed para: (a) secagem a $60^{\circ} \mathrm{C}$; (b) secagem a $70^{\circ} \mathrm{C}$.

Os gráficos gerados pelo Convective são mostrados na Figura 6, em que foram obtidos também o coeficiente convectivo de transferência de massa (h) e o número de Biot. 


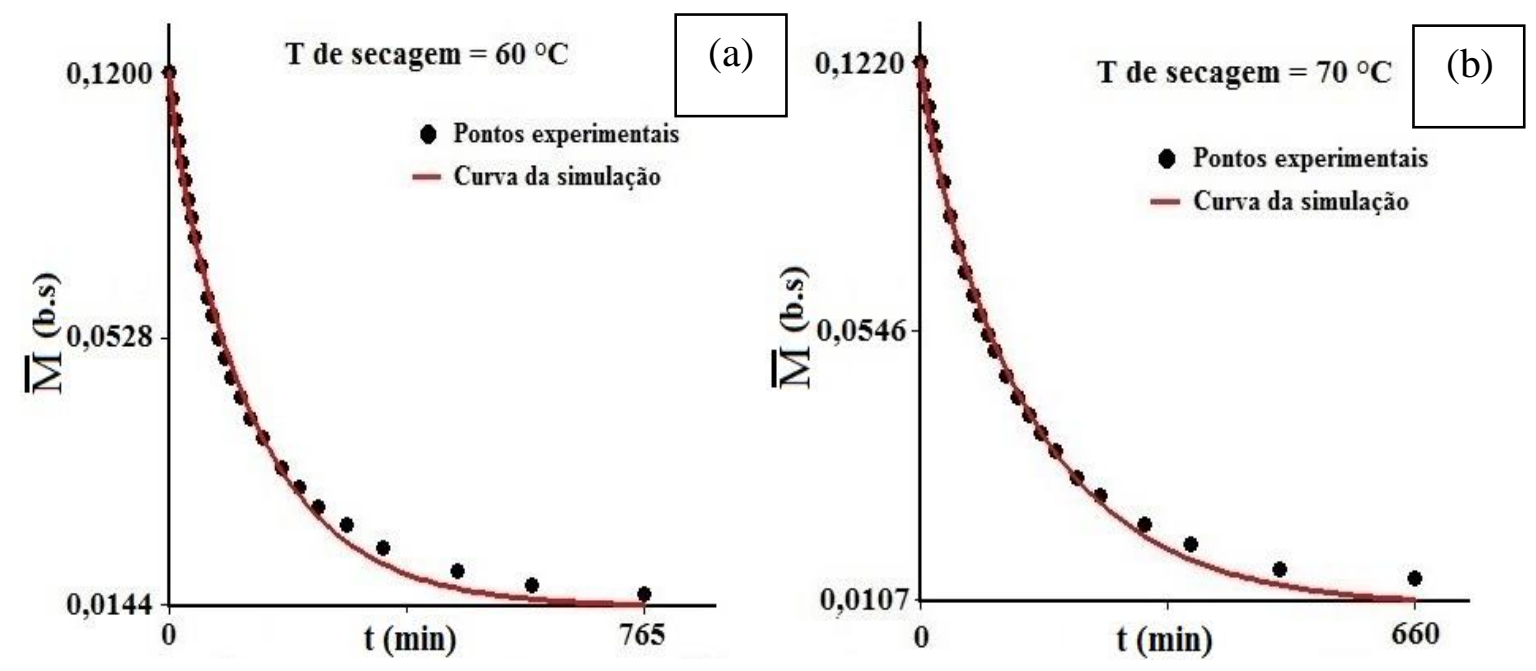

Figura 6: Gráficos das cinéticas de secagem para os períodos de taxa decrescente com pontos experimentais e a curva de secagem simulada pelo software Convective para: (a) secagem a $60^{\circ} \mathrm{C}$; (b) secagem a $70^{\circ} \mathrm{C}$.

Uma inspeção dos gráficos das Figuras 5 e 6 possibilita mostrar que as curvas de secagem simuladas pelos softwares Prescribed e Convective sobrepõem os pontos experimentais obtidos e, que os resultados apresentam indicadores estatísticos $\mathrm{R}^{2}$ e qui-quadrado $\left(\chi^{2}\right)$ com valores bons, visto que quanto mais próximo de 1 é o valor de $\mathrm{R}^{2}$ e menor o valor do $\chi^{2}$, melhor é a aproximação feita pelo algoritmo para o valor da difusividade. Comparando os indicadores estatísticos das simulações realizadas pelos dois softwares é notável que os resultados apresentados pelo Convective são mais consistentes.

Uma característica comum para os resultados obtidos para a Difusividade Mássica através dos dois otimizadores é que seu valor varia com a temperatura do ar de secagem. Quanto maior for a temperatura maior é o valor desse parâmetro.

Assim, espera-se que a secagem a $70 \stackrel{\circ}{\mathrm{C}}$ ocorra de forma mais rápida que a secagem a $60 \stackrel{\circ}{ } \mathrm{C}$.

A Figura 7 apresenta a superposição das cinéticas de secagem para as duas temperaturas estudadas. 


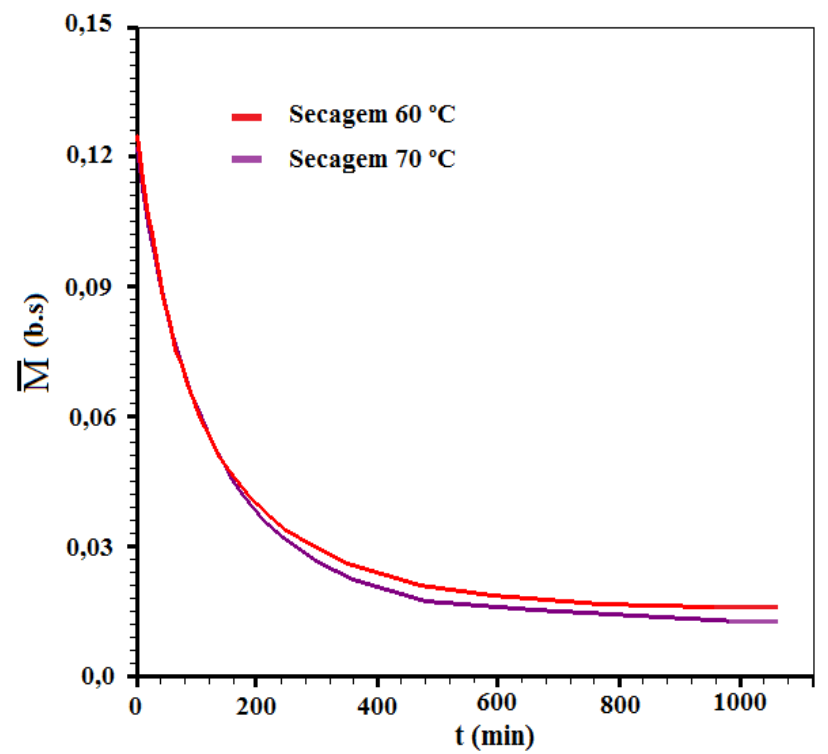

Figura 7: Superposição das cinéticas de secagem para as duas temperaturas.

Uma análise dos gráficos da superposição das cinéticas de secagem apresentados na Figura 7, comprovam que quanto maior é a temperatura de secagem, mais rápido ocorre o fenômeno.

Os resultados permitem concluir que os valores encontrados para a difusividade efetiva de massa $D$ e o coeficiente convectivo de massa $h$ através das otimizações com os softwares Prescribed e Convective são concordantes com outros trabalhos presentes na literatura (FARIAS, 2011; SILVA et al., 2012). Os otimizadores empregados fornecem resultados satisfatórios sem a necessidade do usuário supor valores para as grandezas procuradas, sendo esta uma vantagem dos softwares. Os indicadores estatísticos asseguram valores ótimos para as grandezas procuradas. Nota-se que existe uma variação entre os valores obtidos para $\mathrm{D}$. Isto ocorre porque a condição de contorno de terceira espécie descreve de forma mais realista o processo apresentando valores mais precisos, sendo expresso pelos indicadores estatísticos fornecidos pelo Convective, que são melhores que os fornecidos pelo Prescribed.

\section{Conclusões}

Os dados experimentais permitem identificar dois períodos de secagem: um período com taxa de secagem constante e outro período com taxa decrescente. A transição ocorre em um teor de umidade médio de cerca de 0,12 (b.s.) para as duas temperaturas do ar de secagem. O período de taxa 
constante foi bem descrito por uma equação linear. Já o período de taxa decrescente foi descrito pelo modelo difusivo, onde a equação da difusão unidimensional foi solucionada para condições de contorno de primeiro e terceiro tipo.

As curvas simuladas através da otimização dos softwares Convective e Prescribed se adequaram aos pontos experimentais, explicitando que os valores obtidos para a difusividade são coerentes com o processo, confirmando também a influência da temperatura na rapidez da secagem. A condição de contorno de terceiro tipo (convectiva) se mostrou mais eficiente que a de primeiro tipo (prescrita) para descrever o processo.

Além disso, mesmo considerando que os parâmetros termofísicos são constantes e que as dimensões da telha não variaram durante a secagem, os resultados mostraram que o modelo utilizado foi eficiente na descrição do processo. Desta forma, foi possível concluir que o modelo difusivo (difusão líquida) unidimensional descreve de forma satisfatória a secagem de materiais cerâmicos, no caso, a telha do tipo canal.

\section{Referências}

BACCELLI JÚNIOR, Gilberto. Avaliação do processo industrial da cerâmica vermelha na região do Seridó - RN. 2010. 541 f. Tese (Doutorado em Engenharia Mecânica) - Programa de pós-graduação em Engenharia Mecânica, Universidade Federal do Rio Grande do Norte, Natal, RN, 2010.

BASTOS, Frederico Assis. Avaliação do processo de fabricação de telhas e blocos cerâmicos visando a certificação do produto. 2003. $164 \mathrm{f}$. Dissertação (Mestrado em engenharia civil) - Universidade Federal de Santa Catarina, Florianópolis, SC, 2003.

BIRD, Robert Byron; STEWART, Warren E.; LIGHTFOOT, Edwin N. Transport phenomena. 2nd Ed. New York: John Wiley \& Sons, Inc., 2001.

CHEMKHI, Saber; ZAGROUBA, Féthi. Water diffusion coefficient in clay material from drying data. Desalination, v. 185, p. 491-498, abr. 2005. 
Disponível em: <https://doi.org/10.1016/j.desal.2005.04.052>. Acesso em: 11 abr. 2018.

CRANK, John. The mathematics of diffusion. Oxford, UK: Clarendon Press, 1992.

DINCER, Ibrahim; DOST, Sadik. An analytical model for moisture diffusion in solid objects during drying. Drying Technology, v. 13, n.1-2, p. 425-435, 1995. Disponível em: <http://dx.doi.org/10.1080/07373939508916962>. Acesso em: 9 mai. 2018.

DONDI, Michele; RAIMONDO, Mariarosa; ZANELLI, Chiara. Clays and bodies for ceramic tiles: Reappraisal and technological classification. Applied Clay Science, v. 96, p. 91-109, 2014. Disponível em: <https://doi.org/10.1016/j.clay.2014.01.013>. Acesso em: 9 mai. 2018.

FARIAS, Vera Solange de Oliveira et al. Assessment of diffusion models to describe drying of roof tiles using generalized coordinates. Heat Mass Transfer, v. 52, n. 7, p. 1403-1416, 2015. Disponível em: <https://doi.org/10.1007/s00231-015-1663-7>. Acesso em: 11 abr. 2018.

FARIAS, Vera Solange de Oliveira. Difusão 3D em sólidos com forma arbitrária usando coordenadas generalizadas. 2011. 265 f. Tese (Doutorado em Engenharia de Processos) - Programa de Pós-Graduação em Engenharia de Processos, Universidade Federal de Campina Grande, Campina Grande, PB, 2011.

FARIAS, Vera Solange de Oliveira et al. Drying study of ceramic tiles using Three-dimensional analytical solution of the diffusion equation. JP Journal of Heat and Mass Transfer, v. 15, n. 2, p. 409-432, 2018. Disponível em: <http://dx.doi.org/10.17654/HM015020409>. Acesso em: 11 abr. 2018.

FARIAS, Vera Solange de Oliveira et al. Drying of solids with irregular geometry: numerical study and application using a three-dimensional model. Heat Mass Transfer, v. 49, p. 695-709, jan. 2013. 
FARIAS, Vera Solange de Oliveira et al. Simulação do processo de secagem de telhas cerâmicas através do modelo de difusão usando soluções analíticas para diferentes geometrias. In: XVIII CONGRESSO BRASILEIRO DE ENGENHARIA QUÍMICA, 2010. Anais do XVIII Congresso brasileiro de engenharia química. Foz do Iguaçu: XVIII COBEQ, 2010.

INCROPERA, Frank. P. et al. Fundamentos de transferência de calor e de massa. Rio de Janeiro: LTC, 2008.

LIMA, Antônio Gilson Barbosa de et al. Clay Products Convective Drying: Foundations, Modeling and Applications. Drying and Energy Technologies, Advanced Structured Materials, v. 63, 2016. Disponível em: <https://doi.org/10.1007/978-3-319-19767-8_3>. Acesso em: 11 abr. 2018.

LUIKOV, Aleksei Vasilievich. Analytical heat diffusion theory. London: Academic Press, Inc. Ltd, 1968.

PARK, Kil Jin et al. Conceitos de processo e equipamentos de secagem. Campinas, 121 p., Março de 2007.

PERDONÁ, Camila. Análise do processo de secagem de pasta de cerâmica vermelha utilizando planejamento experimental estatístico. 2002. $16 \mathrm{f}$. Trabalho de Conclusão de Curso (Curso de Tecnologia em Cerâmica) Universidade do Extremo Sul Catarinense, 2012.

SANDER, Aleksandra; SKANSI, Darko; BOLF, Nenad. Heat and mass transfer models in convection drying of clay slabs. Ceramics International, v. 29, n. 6 , p. 641-653, 2003. Disponível em: <https://doi.org/10.1016/S02728842(02)00212-2>. Acesso em: 11 abr. 2018.

SILVA, Laerson Duarte et al. Estudo da secagem de placas cerâmicas de revestimento através do modelo difusivo usando soluções analíticas para condição de contorno do terceiro tipo. In: VII CONGRESSO NACIONAL DE ENGENHARIA MECÂNICA, 2012. Anais do VII Congresso Nacional de Engenharia Mecânica. São Luis: CONEM 2012, 2012. 
SILVA, Wilton Pereira da et al. Drying of clay slabs during the falling rate period: optimization and simulation of the process using diffusion models. Journal of Materials Science Research, v. 2, n. 2, 2013.

SILVA, Wilton Pereira da et al. Estudo da cinética de secagem de telhas cerâmicas através do modelo de difusão usando solução analítica e método inverso. In: CONGRESSO BRASILEIRO DE SISTEMAS PARTICULADOS, 2009. Anais do $34^{\circ}$ Congresso Brasileiro de Sistemas Particulados. Campinas: XXXIV ENEMP, 2009.

SILVA, Wilton Pereira da; e SILVA, Cleide Maria Diniz Pereira da Silva. "Convective Adsorption-Desorption". Versão 2.5 (2009a), online, disponível em: $<$ http://zeus.df.ufcg.edu.br/labfit/Convective.htm>, data de acesso: 22 mar. 2018.

SILVA, Wilton Pereira da; e SILVA, Cleide Maria Diniz Pereira da Silva. "LAB Fit Curve Fitting Software". Versão 7.2.49 (2009b), online, disponível em: $<$ www.labfit.net>, data de acesso: 22 mar. 2018.

SILVA, W.P. e SILVA, C.M.D.P. "Prescribed Adsorption-Desorption". Versão 2.2 (2008), online, disponível em: http://zeus.df.ufcg.edu.br/labfit/Prescribed.htm, data de acesso: 22 mar. 2018. 